\title{
EDITORIAL
}

\section{POLÍTICAS PÚBLICAS PARA LA PROMOCIÓN DE LA ALIMENTACIÓN SALUDABLE Y LA PREVENCIÓN DE LA OBESIDAD}

\author{
Félix Lobo $(1,2)$
}

(1) Agencia Española de Seguridad Alimentaria y Nutrición. Ministerio de Sanidad y Consumo.

(2) Departamento de Economía Aplicada. Universidad Carlos III de Madrid.

La evolución de la obesidad es una preocupación creciente de las autoridades sanitarias nacionales e internacionales. Lo es por la ascendente evolución de su frecuencia, porque constituye en sí una enfermedad, por ser un factor de riesgo de otras enfermedades graves (diabetes, enfermedades cardiovasculares, artrosis, cáncer...), porque pone en peligro la viabilidad de los sistemas sanitarios al causar pesados costes económicos directos e indirectos, porque también, y muy en primer término, afecta a los niños y a los jóvenes y porque nadie debiera esperar la solución de este problema o, ni siquiera, un punto de inflexión para sus tendencias, a menos de diez años vista.

Expresión de esta preocupación son los importantes documentos elaborados por organismos nacionales e internacionales en años recientes. Mencionemos La Estrategia Global sobre Dieta, Actividad Física y Salud de la OMS (aprobada por Resolución de su Asamblea WHA57.17) ${ }^{1}$; la Carta Europea contra la Obesidad ${ }^{2}$, el Libro Blanco de la Unión Europea ${ }^{3}$; el reciente informe de FORESIGHT UK (2007) $)^{4}$ y el documento base de la Estrategia NAOS española 5 .

Existen ya algunos estudios que recogen los preocupantes datos del problema de la obesidad en nuestro país y de sus consecuencias. Mencionemos aquí sólo algunos hallazgos derivados de un estudio reciente desarrollado manejando una base de datos distinta de las que se suele emplear en los estudios epidemiológicos ${ }^{6}$. Se trata del European Community Household Panel de EUROSTAT. Los países europeos estudiados son nueve (Italia, Dinamarca, Bélgica, Irlanda, Grecia, España, Portugal, Austria y Finlandia), entre 1998 y 2001. Los datos son para adultos entre 15 y 75 años. En ellos, con la excepción de los tres primeros, en el año 2001 por lo menos el 50\% de los hombres serían pre-obesos (IMC superior a 25 y menor de 30) o bien obesos (IMC superior a 30). Lo mismo ocurre con el $25 \%$ de las mujeres en todos los países considerados. España tiene, con Finlandia, la tasa de obesidad más alta (13\%) para las mujeres y, esta vez en solitario, la tasa más alta para los hombres $(13,9 \%)$.

Uno de los hallazgos más importantes del trabajo es la alta tasa a la que está creciendo a lo largo del tiempo la epidemia de obesidad: un $8,5 \%$ de media para todos los países considerados en un periodo de sólo cuatro años. (10\% para las mujeres y $8,5 \%$ para los hombres en España). Todos estos resultados vienen a ratificar, con una nueva y solvente fuente, los motivos de preocupación que ya teníamos

La OCDE está insistiendo en sus informes más recientes en que la obesidad es 
uno de los más importantes factores de riesgo que ponen en peligro la salud y que mayores repercusiones va a tener en el futuro sobre el crecimiento del gasto sanitario. La OCDE ha destacado que en EE.UU. los costes de asistencia sanitaria son un $36 \%$ mayores y los de la medicación un $77 \%$ más altos para las personas obesas ${ }^{7}$. Estas diferencias se dan también en los países europeos. El crecimiento de la obesidad en las dos décadas pasadas en la mayor parte de los países de la OCDE se traducirá en mayores costes sanitarios en el futuro. $\mathrm{Su}$ estudio reciente sobre previsiones de gasto sanitario dedica todo un capítulo a la obesidad. "Si no se controla, esta enfermedad puede erosionar en un futuro cercano los avances en longevidad saludable logrados hoy para la gente mayor, al tiempo que supondrá una carga adicional en los costes sanitarios globales"8.

En febrero de 2007 se cumplieron dos años desde el lanzamiento por el Ministerio de Sanidad y Consumo a través de la Agencia Española de Seguridad Alimentaria de la Estrategia por la Nutrición, la Actividad física, contra la Obesidad y por la Salud, o Estrategia NAOS. Puede afirmarse que la Estrategia NAOS ha tenido un éxito y una acogida notables. Este éxito inicial ha consistido básicamente en haber logrado unos niveles de sensibilización y concienciación de muy diversos sectores sociales en un plazo de tiempo verdaderamente corto. También se debe a la puesta en marcha de ciertos programas y acciones concretos de los que se da cuenta en este monográfico. Estos logros derivan, sin duda, de la implicación en la estrategia de esos mismos sectores sociales, reflejada no sólo en declaraciones programáticas de cara a la galería sino en la aceptación de ciertos compromisos que están empezando a ser cumplidos. La Estrategia se ha visto pues catapultada por el potente resorte que puede llegar a suponer la cooperación de los sectores privados con el sector público cuando sus intereses no están contrapuestos.
Una de las grandes tareas que nos espera es desarrollar todas las dimensiones de la Estrategia en programas de acción detallados y con los recursos pertinentes. Tengo que decir que el Ministerio está haciendo un esfuerzo para dotarla con personal y financiación a su servicio.

Un acontecimiento de gran valor simbólico y jurídico ha sido la modificación, por unanimidad de todos los grupos parlamentarios, de la Ley 11/2001, de 5 de julio, que creó la Agencia Española de Seguridad Alimentaria ${ }^{9}$, en virtud de la disposición final octava de la Ley 44/2006 de 29 de diciembre de mejora de la protección de los consumidores y usuarios ${ }^{10}$. La ley ha cambiado el nombre de la Agencia añadiendo al mismo la Nutrición y atribuyéndole, con el máximo rango normativo, la responsabilidad de planificar, coordinar y desarrollar estrategias y actuaciones que fomenten la información, educación y promoción de la salud en el ámbito de la nutrición y en especial la prevención de la obesidad. También la dota de algunos instrumentos legales para mejorar su capacidad de actuación (legitimación activa para interponer acciones de cesación). La razón de estas modificaciones estriba en la necesidad de superar un concepto anticuado de la salud alimentaria, restringido a los aspectos de mera protección de la salud frente a las enfermedades con origen en el consumo de productos alimenticios, y entra de lleno en la promoción de la salud mediante la mejora de las condiciones de vida y la actividad física. Son éstos refuerzos normativos que facilitarán y darán continuidad a las tareas de la Agencia. Seguimos así, por cierto, una tendencia europea en la reorganización de las administraciones públicas sanitarias encargadas de la seguridad alimentaria, consistente en añadir a sus responsabilidades la de afrontar la tendencia creciente de la obesidad.

¿Cuáles son los rasgos generales que han caracterizado el desarrollo de la Estrategia 
NAOS en este periodo? Podríamos destacar los siguientes:

La Estrategia NAOS tiene para el Gobierno español una muy alta prioridad política. El propio Presidente del Gobierno así lo expresó en su discurso sobre el Estado de la Nación ante el Congreso de los Diputados el día 30 de mayo de 2006, al anunciar como uno de sus programas destacados en sanidad la lucha contra la obesidad infantil.

La Estrategia pretende tener en sus iniciativas el más sólido apoyo científico. Desde el principio un elenco de grupos de trabajo formados por destacados científicos, clínicos y otros expertos ha colaborado en su formulación y desarrollo. Un fruto destacado ha sido la publicación del libro que recopila los informes técnicos de dichos grupos ${ }^{11}$.

La colaboración interinstitucional e intersectorial, recogidas como deberes para la AESAN en su ley fundadora, caracterizan también a la Estrategia NAOS. La función de las comunidades autónomas y los municipios en la difusión y promoción de las condiciones para una alimentación saludable y de la actividad física es de la máxima importancia. Colaborar con todas las entidades territoriales es un eje insoslayable de nuestra política marcado por la naturaleza de nuestra Constitución. Un ejemplo lo constituye el Programa PERSEO, de promoción de los hábitos alimentarios saludables y la actividad física en las escuelas, iniciado el año 2006 en unión con el Ministerio de Educación y en el que participan en ésta su primera fase seis comunidades autónomas, del que se trata en este monográfico ${ }^{12}$. Hay que decir a este respecto, que existe ya entre comunidades autónomas y municipios un sano espíritu de emulación por proporcionar a los ciudadanos y especialmente a nuestros niños los mejores programas de prevención de la obesidad.
En cuanto a la cooperación intersectorial la estrategia NAOS se ha distinguido por intentar movilizar a los más diversos sectores y actores sociales, entre ellos el mundo empresarial. La industria alimentaria y las empresas de distribución han mantenido una posición muy positiva respecto de la estrategia y han firmado convenios con compromisos de gran alcance. Esta dimensión ha sido internacionalmente reconocida, como veremos a continuación.

Podríamos decir también que la presencia constante de la Estrategia NAOS en los medios de comunicación es otra de sus características. Así, uno de sus objetivos, sensibilizar a la población acerca de la trascendencia sanitaria del problema de la obesidad, está ya más próximo, pues los medios de comunicación en la sociedad moderna son un canal fundamental para la obtención de información y el cambio de comportamientos por los ciudadanos.

España mantiene un lugar destacado entre los países a los que se atribuye un mayor dinamismo al enfrentarse al reto de contrarrestar la epidemia de la obesidad como expresan los reconocimientos internacionales de la Estrategia NAOS. Un jurado, reunido por la Oficina Regional Europea de la Organización Mundial de la Salud en su Conferencia Ministerial celebrada en Estambul el mes de noviembre de 2006, otorgó a la Estrategia NAOS uno de sus premios, por la forma en que aborda la colaboración entre las administraciones públicas y los agentes sociales privados. La Unión Europea también nos ha hecho reiteradamente el honor de invitar al Ministerio y a la Agencia Española de Seguridad Alimentaría y Nutrición como ponente a sus distintos foros para explicar nuestras experiencias en el desarrollo de la Estrategia. También ha reconocido su interés la oficina central de Ginebra de la OMS que organizó en Madrid, con la colaboración del Ministerio y la Agencia, una reunión técnica sobre indicadores de seguimiento de los progra- 
mas de prevención de la obesidad en octubre de 2007.

La estrategia NAOS ha de incorporar la perspectiva de género porque, por un lado, la obesidad, en ciertas dimensiones, afecta más intensamente a las mujeres y porque, por otro lado, el protagonismo de la mujer en la selección y en la preparación de los alimentos en los hogares españoles es abrumador. En efecto, la obesidad afecta especialmente a las mujeres, en particular a partir de cierta edad. Entre las personas mayores de sesenta años hay más hombres con sobrepeso $(49 \%$ frente a $39,8 \%$ en las mujeres) pero hay más mujeres con obesidad, que es la situación más preocupante. Según su IMC, frente a un $31,5 \%$ de hombres obesos hay un $40,8 \%$ de mujeres obesas. Si en lugar del IMC consideramos la "obesidad central", es decir la que toma como parámetro de medida la circunferencia de la cintura, hay $78,4 \%$ de mujeres obesas frente a $48,4 \%$ de hombres ${ }^{13}$. Estos datos, confirmados por otros muchos, ponen de relieve la necesidad de desarrollar programas de prevención y tratamiento de la obesidad específicos para mujeres.

Por otro lado, sabemos que mayoritariamente son las mujeres las que hacen la compra en el mercado y las que cocinan. Según la última Encuesta sobre usos del tiempo, que es de 2001, las mujeres dedican al trabajo de la casa cinco veces más tiempo que los hombres y el doble de tiempo a las compras ${ }^{14}$. La realidad es pues que las mujeres tienen un gran protagonismo en la decisión de optar por unos u otros hábitos alimentarios que luego sigue toda la población y muy en particular los niños. Por ello las actividades de sensibilización, información y educación de la estrategia NAOS van a tener muy en cuenta que las mujeres deben constituir un objetivo privilegiado, al tiempo que propiciarán un mayor equilibrio en la distribución de estas tareas.
Finalmente quiero destacar que la estrategia NAOS tiene una dimensión de equidad o justicia distributiva respecto de los menos favorecidos. Las personas y los grupos sociales menos favorecidos en términos de renta y de educación y cultura sufren más la enfermedad de la obesidad y tiene menos acceso a alimentos de más calidad, a información solvente sobre hábitos alimentarios y al ejercicio físico. Como dice el Informe Técnico General de la Estrategia "en los países desarrollados la prevalencia de obesidad es mayor en los grupos socioeconómicos más deprimidos" "11. Según Sanz de Galdeano 6 "la prevalencia de la obesidad está claramente asociada con el estatus socioeconómico (definido por educación y renta familiar) bajo" (aunque no encuentra asociación estadísticamente significativa para la renta en el caso de los hombres). "Hombres y mujeres con sólo educación primaria tiene una probabilidad sustancialmente mayor de ser obesos que los que tiene educación universitaria". Por ello la estrategia NAOS también va a considerar que estos grupos merecen una atención especial en sus programas, particularmente de los que pretenden sensibilizar, informar y educar.

Concluyo con palabras de la Ministra de Sanidad y Consumo que también hago mías: "mi agradecimiento a las decenas de científicos, clínicos y expertos de todo tipo, sociedades científicas, municipios, asociaciones, empresas, medios de comunicación y entidades diversas que están contribuyendo al desarrollo de la estrategia NAOS contra la obesidad. A pesar de su gran esfuerzo la tarea es tan grande que sólo hemos avanzado los primeros pasos. Por eso tengo nuevamente que convocarles a todos a seguir colaborando y prestando su apoyo. Necesitamos una auténtica movilización social en pro de la Estrategia NAOS contra la obesidad y en interés de la salud de todos los españoles, especialmente de los que hoy son niños o jóvenes pero que pronto serán los adultos que habrán de "hacer país" cuando nosotros ya nos retiremos." 


\section{BIBLIOGRAFÍA}

1. OMS. Estrategia Global sobre Dieta, Actividad Física y Salud, Ginebra: OMS; 2004.

2. OMS. Carta Europea contra la Obesidad. Ministerial Conference on Counteracting Obesity. Istanbul, Turkey on 15-17 November; 2006.

3. Comisión Europea. Libro Blanco sobre la Obesidad. Bruselas: Comisión Europea; 2007.

4. Eastern Region Public Health Observatory. Tackling Obesity: Future Choices - Foresight Report. Cambridge; 2007.

5. Agencia española de seguridad alimentaria (AESA): Estrategia NAOS. Estrategia para la Nutrición, Actividad Física y Prevención de la Obesidad. Ministerio de Sanidad y Consumo; Madrid; 2005.

6. Sanz de Galdeano A. The Obesity Epidemic in Europe. Forschunginstitut zur Zukunf der Arbeit (IZA). October, Bonn, Discussion Paper No 1814; 2005.

7. OCDE. Health at a Glance. OCDE Indicators. París: OCDE; 2005.

8. OCDE. Previsiones de gasto sanitario de la OCDE 2006. París: OCDE; 2006.
9. Boletín Oficial del Estado. Ley 11/2001, de 5 de julio, por la que se crea la Agencia Española de Seguridad Alimentaria. BOE num 161 de 06/07/01.

10. Boletín Oficial del Estado. Ley 44/2006, de 29 de diciembre, de mejora de la protección de los consumidores y usuarios. BOE num 312 de 30/12/2006.

11. Moreno B, y Charro A eds. La Estrategia NAOS. Madrid: AESAN y Editorial Panamericana; 2006.

12. Ballesteros Arribas JM, Dal-Re Saavedra M, Pérez FarinósN, Villar Villalba C. La estrategia para la nutrición, actividad física y prevención de la obesidad (estrategia NAOS). Rev Esp Salud Pública 2007; 81:443-49

13. Gutiérrez-Fisac JL, López E, Banegas JR, Graciani A, Rodríguez-Artalejo F. Prevalence of Overweight and Obesity in Elderly People in Spain. Obes Res 2004; 12: 710-5.

14. Agencia española de seguridad alimentaria y nutrición (AESAN): Estrategia NAOS. Actividades y Proyectos. Primavera 2007. Madrid: Ministerio de Sanidad y Consumo; 2007.

15. Instituto de la mujer. Encuesta sobre los usos del tiempo 2001. Madrid: Instituto de la mujer; 2002. 
